\title{
Capital social y los resultados de los grupos de investigación, desarrollo tecnológico e innovación del departamento del Atlántico, Colombia
}

\author{
Karelis del C. Barrios-Hernández ${ }^{1^{*}}$, Pedro García-Villaverde ${ }^{2}$ y María J. Ruiz-Ortega ${ }^{2}$ \\ (1) Facultad de Administración y Negocios, Universidad Simón Bolívar, Barranquilla-Colombia \\ (correo-e: kbarrios22@unisimonbolivar.edu.co; karelisbarrios03@gmail.com). \\ (2) Escuela Internacional de Doctorado en Economía y Empresa. Universidad Castilla La Mancha, Albacete- España \\ (correo-e: Pedro.GVillaverde@uclm.es; MariaJose.Ruiz@uclm.es).
}

* Autor a quien debe dirigirse la correspondencia

Recibido Jun. 23, 2020; Aceptado Ago. 25, 2020; Versión final Nov. 16, 2020, Publicado Feb. 2021

\begin{abstract}
Resumen
El objetivo del presente estudio es determinar la relación entre el capital social (bonding y bridging) y los resultados de los grupos de investigación, desarrollo tecnológico e innovación de las instituciones de educación superior (IES) en el Departamento del Atlántico, Colombia. Se analizó la literatura existente (Scielo, Scopus, Elsevier y Emerald) entre el año 2010 y 2020. Se compararon los referentes bibliográficos de capital social con los indicadores del perfil de colaboración de los grupos de investigación categorizados por el Ministerio de Ciencia y Tecnología del Departamento del Atlántico. Los principales hallazgos destacan que las relaciones que los investigadores establecen, permiten generar valor institucional porque promueven el aprendizaje y responden a necesidades del entono. Se concluye que siendo las IES generadoras de nuevo conocimiento, parte de sus resultados se ven representados por la relación interna y externa de los grupos de investigación, los cuales facilitan y fortalecen el trabajo en la medida que establecen conexiones de cohesión y cooperación.
\end{abstract}

\section{Social capital and results published by research, technological development, and innovation groups from Atlántico state, Colombia}

\begin{abstract}
The objective of the present study is to determine the relationship between social capital (bonding and bridging) and results published by research, technological development, and innovation groups at higher education institutions (HEls) from the state of Atlántico, Colombia. A literature review (Scielo, Scopus, Elsevier, and Emerald) between the years 2010 and 2020 is performed. Social capital references are compared to collaboration profile indicators for research groups ranked by the Ministry of Science and Technology from the state of Atlántico. The results show and highlight that the relationships that researchers establish allow generating institutional value since they promote learning and respond to surrounding needs. It is concluded that as HEls generate new knowledge, a part of this can be seen represented by their research groups' internal and external relationships, which facilitates and strengthens their work as they establish cohesive and collaborative connections.
\end{abstract}




\section{INTRODUCCIÓN}

Las organizaciones requieren actualmente adaptarse y responder con productos y servicios ajustados a las necesidades del mercado, debido a que están sumergidas en entornos de cambios rápidos representados por las situaciones políticas, económicas, legales, sanitarias y medioambientales que vive el mundo. Esta situación no es ajena en las Instituciones de Educación Superior (IES). Por una parte, son instituciones cuyo rol como actor de la sociedad ha cambiado en la última década, dando paso a nuevas actividades que han reconfigurado su razón de ser, contribuyendo a resolver la problemática local, regional y nacional (BarriosHernández et al., 2017). Adicionalmente, aplican y divulgan su saber hacer a través de resultados de investigación, desarrollo tecnológico e innovación ( $1+D+i)$, que demuestran la dinámica de interacción de la academia, la empresa, el estado y la sociedad (Barrios-Hernández y Olivero-Vega, 2020; Barrios-Hernández, et al., 2020)

Por otra parte, las IES están inmersas en el Sistema Nacional de Ciencia, Tecnología e Innovación (SNCTel), en el que se evidencia una amplia heterogeneidad en los resultados de itnvestigación entre grupos, vinculada a la forma en que responden a las condiciones cambiantes del entorno. Por eso, la investigación cumple un doble rol. El primero relacionado con la generación de nuevo conocimiento para entender la realidad de la sociedad. El segundo es un proceso integrador de las relaciones que establece con la academia, la universidad y el estado, para generar resultados que respondan a las necesidades del entorno y al aprovechamiento de las oportunidades.

En Colombia, las IES generan resultados de investigación a través de sus grupos. Se entiende como grupo de investigación, desarrollo tecnológico o de innovación a la unión de un grupo de personas que se integran para el desarrollo de investigaciones y generan productos de conocimiento en una o varias temáticas (Ministerio de Ciencia y Tecnología, 2018). Para el reconocimiento como grupo de investigación, este debe demostrar continuamente resultados verificables, derivados de proyectos y de otras actividades y que, además, cumpla con los requisitos mínimos de calidad para su reconocimiento. En Colombia se encuentran clasificados en orden descendente en A1, A, B y C. Esta categorización se alcanza acorde a la tipología, visibilidad e impacto de los productos de investigación, desarrollo tecnológico e innovación registrados.

En la última medición de grupos de investigación, desarrollo tecnológico e innovación y en el reconocimiento de investigadores del SNCTel realizada en el año 2018, se evidenció la categorización de 16.799 investigadores, con la siguiente distribución: investigadores eméritos: 56; investigadores sénior: 2.473; investigadores asociados: 4.349; e investigador junior: 9.921. En cuanto al reconocimiento y medición de grupos de investigación, se recogen 5.772 registrados en la plataforma GrupLAC, que fueron avalados por, al menos, una institución. De estos registros de grupos, 5.589 fueron reconocidos como grupos de investigación, desarrollo tecnológico e innovación y 5536 fueron categorizados, y la distribución de éstos es la siguiente: 717 Grupos A1; 1.023 Grupos A; 1.285 Grupos B; y 2.328 Grupos C y 236 reconocidos que no alcanzaron categorización (Ministerio de Ciencia y Tecnología, 2019). A nivel nacional, al comparar las dos ultimas convocatorias de medición, se evidencia un incremento del $10,85 \%$ en el número de grupos. Específicamente en el departamento del Atlántico se observa un crecimiento de los grupos de investigación en categoría A1 y A del 26,5\%. Esta situación se presenta muy a pesar que a nivel nacional la tendencia de inversiones en actividades de ciencia, tecnología e innovación no ha sido la mejor, con una variación del $11,59 \%$. Sin embargo, se presenta un aumento del $8,13 \%$ en las actividades de investigación y desarrollo entre los años 2017 y 2018.

En este trabajo se aborda el constructo capital social y su relación con los resultados de los grupos de investigación. El concepto de capital social es entendido como un activo intangible representado en el conjunto o redes de relaciones de alto valor que generan los individuos dentro o fuera de un grupo, con el fin de satisfacer no solo sus necesidades individuales y grupales, sino también para la generación de ventajas a través del aprovechamiento de oportunidades (Coleman, 1988). A partir de este concepto, siguiendo a Putnam (2000), se distinguen dos dimensiones del capital social, el capital social bonding y el capital social bridging.

El capital social bonding proviene de redes densas y cercanas donde los lazos fuertes dan forma a la confianza, así como a los valores y objetivos compartidos. Se basa en relaciones entre individuos con características comunes o pertenecientes al mismo colectivo o territorio, es decir se enfoca en las relaciones internas que posee un integrante del grupo, estimulando la transferencia de conocimiento y la protección en entornos interorganizacionales (Dyer y Nobeoka, 2000; Kale et al., 2000). Es así como, el capital social bonding, abarca la interacción social, vínculos fuertes y las redes densas, lo que se convierte en un determinante clave para el intercambio de conocimiento (Elche-Hortelano et al., 2015).

Por su parte, el capital social bridging se construye sobre lazos débiles y agujeros estructurales, que facilitan el acceso a nueva información. Se centra en las relaciones entre individuos con características diferentes o pertenecientes a colectivos o territorios diferentes, es decir, su enfoque son las relaciones externas 
(Lozares et al., 2011). Por tanto, el capital social bridging se refiere a la conexión, producida entre redes diversas (Putnam, 2000), que vinculan a los actores de grupos diferentes para facilitar descubrimiento de oportunidades y el acceso a información nueva y diferente, a través del refuerzo de los lazos sociales por encima de las barreras étnicas, económicas, culturales, sociales o religiosas (Segura, 2011). Estos lazos son muy importantes para el desarrollo de relaciones de valor, ya que permiten la intermediación entre colectivos, y facilitan las alianzas exógenas y el acceso a recursos de los que no dispone el colectivo o comunidad. De esta manera, los individuos o colectivos con elevado capital social bridging influyen decisivamente en el control de los flujos de información y pueden desarrollar posiciones de prestigio o poder (Esparcia et al., 2015). En este trabajo se plantea que ambos pueden afectar de forma significativa a los resultados de los grupos de investigación.

En este sentido, el objetivo de este artículo es revisar los cálculos para cada grupo de investigación, desarrollo tecnológico e innovación en el departamento del Atlántico desde la visión de sus indicadores de capital social, cohesión -bonding capital- y cooperación -bridging capital-, para analizar el trabajo conjunto entre los integrantes del grupo y entre diferentes grupos.

\section{OTROS ANTECEDENTES}

El concepto de capital social está en proceso de construcción, debido a que existen diferentes concepciones sobre el capital social sustentados en diferentes paradigmas teóricos y metodológicos. Algunas perspectivas consideran al capital social como un recurso individual y otras como un atributo de la comunidad, la sociedad o la estructura social (Freyre 2013). Principalmente, los conceptos convergen en que el ser humano es potencialmente sociable y para satisfacer sus necesidades y obtener recursos crea relaciones con otros. Estas relaciones parten del hecho de satisfacer sus demandas desde el deseo de pertenecer a un grupo o conocer personas en diversos ámbitos hasta la consecución de recursos tangibles o intangibles. Inicialmente, el término de capital social fue utilizado para explicar cómo en la satisfacción de las necesidades sociales de los individuos influye el compromiso comunitario. En la Tabla 1 algunas aportaciones adicionales al concepto de capital social.

Tabla 1. Aportaciones del concepto de capital social

\begin{tabular}{|l|l|}
\hline Autor & Definición \\
\hline Bourdieu (1980) & $\begin{array}{l}\text { "Conjunto de recursos actuales o potenciales que están ligados a la posesión de una red } \\
\text { duradera de relaciones más o menos institucionalizadas de interconocimiento y de inter } \\
\text { reconocimiento" Pág. 2 }\end{array}$ \\
\hline $\begin{array}{l}\text { Bourdieu y } \\
\text { Wacquant (1992) }\end{array}$ & $\begin{array}{l}\text { "La suma de los recursos, actuales o virtuales, que se acumulan en un individuo o } \\
\text { unidad, en virtud de poseer una red duradera de relaciones más o menos } \\
\text { institucionalizada de conocidos y de reconocimientos mutuos" Pág. 119 }\end{array}$ \\
\hline Westlund (2006) & $\begin{array}{l}\text { "Redes sociales no formalizadas que son creadas, mantenidas y usadas por los actores } \\
\text { de la red para distribuir normas, valores, preferencias y otros atributos sociales y } \\
\text { características pero que también surgen como resultado de que los actores compartan } \\
\text { algunas de estas características" Pág. 8. }\end{array}$ \\
\hline Martínez (2013) & $\begin{array}{l}\text { Vínculo entre los individuos y la sociedad, considerándolo un recurso a través del cual } \\
\text { los miembros de una red pueden alcanzar diferentes ventajas. }\end{array}$ \\
\hline Arzuero (2009) & $\begin{array}{l}\text { "Reside en las relaciones sociales; suple la carencia de otros recursos en contextos de } \\
\text { pobreza; se fundamenta en la confianza, la reciprocidad, la asociatividad, la acción } \\
\text { colectiva y la cooperación; es un activo intangible que se puede expresar en créditos } \\
\text { económicos". Pág. 155 }\end{array}$ \\
\hline Rostila (2011) & $\begin{array}{l}\text { El capital social se puede definir como los recursos y la confianza integrados en las } \\
\text { redes personales, y se puede acceder y movilizar para lograr objetivos individuales o } \\
\text { colectivos }\end{array}$ \\
\hline
\end{tabular}

Es así como se plantea un paradigma de integración social que señala la importancia de las relaciones sociales en la cooperación social (Forni et al., 2004). En este sentido, se encuentran varias investigaciones sobre el capital social desde cuatro perspectivas diferentes: comunitaria, de las redes, y la sinérgica. La perspectiva comunitaria asocia el capital social con la capacidad de la sociedad de auto-gestionarse a través de organizaciones locales, evidenciando un efecto positivo en el bienestar de la comunidad. Mientras la perspectiva de redes, menciona dos cuestiones. Por una parte, la importancia del fortalecimiento de los lazos intracomunitarios y, por otro, la necesidad de construir lazos intercomunitarios para disminuir barreras internas. A los primeros se les suele llamar capital social bonding y a los segundos, capital social bridging. La tercera perspectiva, la institucional sostiene que la capacidad de los diferentes niveles de grupos sociales para movilizarse por intereses colectivos depende de las instituciones formales con las cuales funcionan. Finalmente, la perspectiva sinérgica unifica la visión que privilegia los ámbitos institucionales con las redes (Capdevielle, 2014). 
Este artículo, se centra desde el paradigma teórico de la visión de redes en el capital social. Autores principales como Bourdieu (1980), Hanifan (1916) y Burt (1992), plantean desde un inicio la importancia de las relaciones. El primero por su parte, mantiene una concepción de capital que es eminentemente relacional en todas sus especies. Desde esta perspectiva teórica el capital social es un recurso que se convierte en una fuente de poder, que se acumula, se invierte, pierde o se reconvierte en otras especies de capital, cuando las condiciones estructurales lo permiten. El segundo manifiesta que los problemas de los individuos podrían resolverse mediante la solidaridad entre ciudadanos, lo que permitía obtener recursos. El tercero, analiza el concepto de capital social dentro de los estudios de redes a través de su teoría de los huecos estructurales, defiende que son estos los que permiten establecer un vínculo puente entre un agente y otros agentes que participan de flujos de información diferentes, gracias a la no redundancia de los vínculos (Putnam, 2000).

Solis \& Limas (2013) establecen que el capital social consiste en un conjunto de relaciones sociales que tiene lugar entre individuos formando una red. La red hace referencia al conjunto de agentes y a la relación o relaciones que los conectan. Por tanto, para el capital social no solo juegan un papel preponderante los individuos que se relacionan, sino la forma como estas relaciones se desarrollan. Es decir, si las relaciones se dan con miembros ajenos al grupo se presenta una red externa y si se produce al interior de la organización se desarrolla una red interna (Walter, Lechner y Kellermanns, 2007). La literatura sugiere que la redes para desarrollarse tienen una estructura, una dinámica y un nivel de calidad propios. Por su parte, la estructura se refiere a la naturaleza de las relaciones, o sea, distingue si la relación es entre los individuos, es de amistad, vecindad, trabajo, negocios, etc. La dinámica por su parte está relacionada con los flujos de recursos tangibles e intangibles que comparten entre las personas. Mientras, la calidad se refiere a la fuerza de las relaciones y a las conexiones de los individuos (Solis y Limas, 2013).

\section{Capital social en el contexto de grupos de investigación}

Para contextualizar este artículo, es importante aproximarnos a diversas investigaciones que permitan conocer la dinámica interna de los grupos de investigación y sus relaciones, que arrojen reflexiones sobre las prácticas de investigación y sobre la identificación de los grupos de investigación como ecosistemas de innovación y conocimiento (Orozco et al., 2013). Ante el proceso de competencia global de la I+D+i en las últimas décadas, la investigación en Colombia debe orientarse a generar innovaciones acordes a las necesidades de un entorno que va desde lo local a lo global. Para ello, los grupos de investigación trabajan, por un lado, en fortalecer la cohesión de las relaciones entre sus integrantes -capital social bonding- y, por otro, en establecer relaciones de cooperación con grupos de investigación diversos y lejanos -capital social bridging- que permitan crear y desarrollar sólidas capacidades. El capital social bonding -cohesión- tiene efectos favorables como la accesibilidad a recursos, o un mayor control en la transferencia de conocimiento.

Adicionalmente, se plantea también, que la continuidad de las relaciones con contactos frecuentes y estrechos podría causar bloqueo y plantear dificultades para el desarrollo de nuevas ideas (Eklinder-Frick et al., 2012). En relación al capital social bridging -cooperación- la literatura resalta su contribución para crear conexiones con agentes externos que contribuyen al desarrollo de nuevas ideas e innovaciones (Doloreux y Mattson 2008). En este contexto, se concibe las IES como organizaciones flexibles que responden de manera coherente, estratégica y oportuna ante los cambios del entorno, generando con sus acciones sostenibilidad en el mercado, sin que ello signifique una amenaza para su misión académica tradicional (Clark, 2004). A partir de esa dinámica de relación, se acerca a los investigadores a las necesidades latentes del entorno en el que se desenvuelven, de tal manera que los proyectos respondan con soluciones prácticas e innovadoras que sin duda alguna mejoran la competitividad de un país (Barrios-Hernández et al.,2017). En este sentido, en el entorno colombiano los grupos de investigación tienen hoy un alto grado de protagonismo para resolver las problemáticas propias de los territorios. Por tanto, es común no solo ver la articulación entre grupos de una misma institución, sino también como realizan trabajos en conjuntos grupos de diferentes Universidades.

Adicionalmente el Ministerio de Ciencia y Tecnología antes denominado Colciencias valora el esfuerzo que realiza cada grupo y su contribución a la generación de productos científicos y tecnológicos. Para ello, dispone cada dos años de una convocatoria que busca medir y categorizar el nivel de producción, la calidad de los mismos y el desarrollo de trabajo colaborativo. De esta manera, cuenta con un modelo de medición de la producción de los grupos de investigación en cuanto a posicionar el conocimiento como un capital relevante para el desarrollo social y económico cuyas capacidades pueden darse paralelamente en lo institucional, relacional o individual. Por tanto, al terminar el proceso de medición se cuenta con un perfil de investigadores y otro de grupos de investigación.

En estos resultados, se obtiene la clasificación de los productos de nuevo conocimiento, desarrollo tecnológico, apropiación social de conocimiento y formación de recurso humano. Los datos correspondientes a la Convocatoria 833 de 2018, se detallan a continuación en la Figura 1, en esta se evidencia que el 49\% fueron producto de nuevo conocimiento, tales como: artículos, libros o capítulos de libro resultado de investigación. El 25\% corresponde con productos de formación del recurso humano, estos son: tutorías de 
trabajo de pregrado, de maestría o tesis doctoral, así como proyectos de investigación, desarrollo e innovación. Mientras el $22 \%$ son productos de apropiación social de conocimiento como consultorías científicas, eventos científicos, estrategias pedagógicas, entre otros. Por último el $4 \%$ están clasificados como de desarrollo tecnológico y algunos de estos productos son innovaciones de procesos y procedimientos, innovaciones de la gestión empresarial, patentes, signos distintivos, modelos de utilidad, etc.

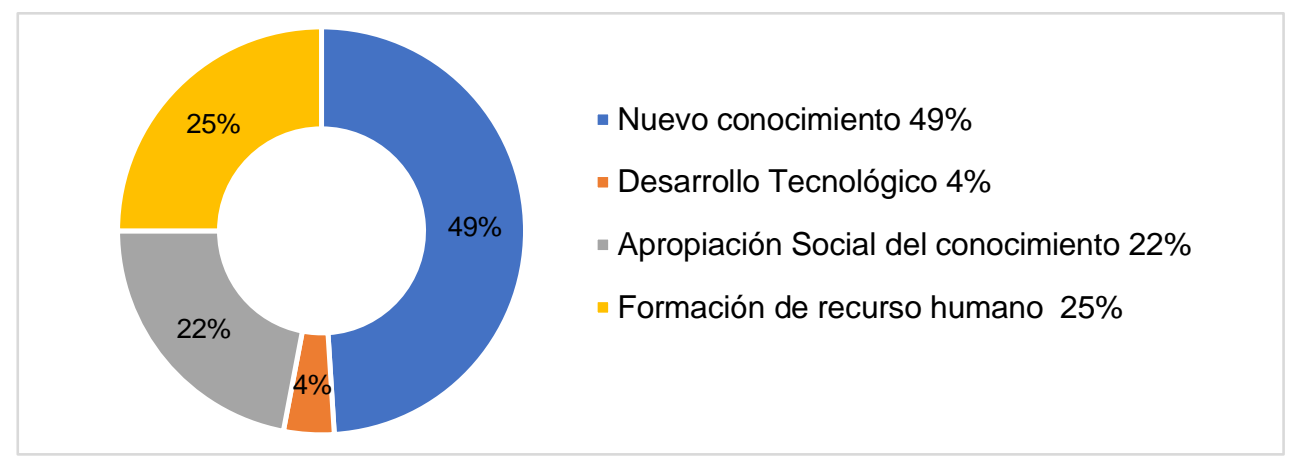

Fig. 1: Producción general de grupos de investigación en Colombia

El Ministerio de Ciencia y Tecnología no solo mide la cantidad y calidad de productos, sino que además establece como se logra esta producción en los grupos y entre grupos, midiendo de esta manera el capital social de ellos. En el contexto de universidades se evidencia que sobre capital social en grupos existen diferencias entre campos académicos, tal como lo presenta Kyvik \& Reymert (2017) en las cuatro principales universidades de investigación de Noruega, quienes concluyeron que, ocho de cada diez académicos forman parte de un grupo de investigación formal. Sin embargo, la tasa de participación varía acorde al campo de estudio, los porcentajes más altos están en las ciencias naturales y ciencias de la salud, específicamente en el programa de medicina. La más baja participación se demostró en las humanidades, donde solo seis académicos de cada diez son miembros. Finalmente, demostraron que ser profesor incrementa la probabilidad de realizar trabajos conjuntos en una red internacional y disminuye la probabilidad de trabajar solo. Aunque ser miembro de un grupo de investigación no significa necesariamente que el personal académico realiza su investigación en gran medida dentro del grupo, ser miembro aumenta la probabilidad de hacerlo. Ser miembro de un grupo también aumenta la probabilidad de trabajar en una red internacional en combinación con un grupo, y disminuye la probabilidad de trabajar solo.

Por otro lado, la investigación realizada por Gaette (2008) presenta un enfoque de relaciones sociales en el campo de la educación superior, a partir de la identificación y análisis de las redes de conocimiento recreadas desde la dinámica de las relaciones que establecen los investigadores ligados al desarrollo de las ciencias sociales y sus grupos de investigación. Los resultados más relevantes se asocian con que la posición académica de los investigadores que conforman una red, no determina, ni asegura, un posicionamiento relevante en una red donde circulan intangibles tan valiosos como la información y el conocimiento.

Desde otra perspectiva Steinmo y Rasmussen (2018) presentan que la dinámica organizacional que subyace a las relaciones externas exitosas entre la universidad y la industria en el proceso de innovación se considera un importante impulsor de la competitividad de las empresas. Sin embargo, estas relaciones son poco conocidas. Mediante estudios de 15 proyectos de innovación, examinaron cómo las empresas con experiencia en colaboración con universidades y organizaciones de investigación públicas confían en diferentes dimensiones de capital social para lograr colaboraciones exitosas. En sus resultados más importantes, encontraron que las empresas experimentadas establecen colaboraciones externas sobre la base del capital social cognitivo, (basado en los objetivos, metas y cultura compartidas), reforzada por el capital social relacional (basado en las relaciones de confianza) a lo largo del tiempo (Nahapiet y Goshal, 1998). Por tanto, parte de su éxito se fundamenta en la experiencia pasada generada y en la base de sus relaciones efectivas. Por el contrario, las empresas con menos experiencia basan sus colaboraciones universitarias en el capital social relacional, que se ve reforzado por el capital social cognitivo a lo largo del tiempo. De esta manera, los autores muestran que las dimensiones cognitivas y relacionales del capital social son relevantes para una colaboración exitosa. No obstante, la principal aportación de los investigadores consiste en que estas dimensiones de capital social interactúan y se desarrollan con el tiempo en función del nivel inicial de capital social cognitivo de la empresa generado por la experiencia previa con colaboraciones universitarias y experiencia académica. Sin embargo, la forma en que las empresas con poca experiencia previa en colaboración universitaria y experiencia académica demuestra que estas suelen confiar en las relaciones individuales cuando establecen colaboraciones.

Por otra parte, en un estudio realizado sobre el uso de un marco de capital social para apoyar a los grupos de estudiantes de doctorado, en la cual cuatro graduados de doctorado participaron en un grupo de redacción, 
en el que examinaron la influencia del capital social, descubrieron que un fuerte factor social respaldaba sus esfuerzos para participar como académicos, ser responsables de sus escritos y hacer contribuciones a la comunidad investigadora. Las estrategias para fomentar la generación de investigaciones se proporcionan utilizando las tres dimensiones del capital social: estructural, relacional y cognitivo (Tyndall et al., 2019).

Adicionalmente, Barletta et al., (2017) en un estudio en 314 grupos de investigación de Argentina en tecnología de la información y comunicación (TIC), exploraron sobre la productividad científica y las actividades de transferencia. En primer lugar, indagaron a cerca de los factores relacionados con el desempeño de los grupos de investigación en términos de transferencia de tecnología y productividad científica. En segundo lugar, analizaron la relación entre la productividad científica de los grupos y su rendimiento en la transferencia de tecnología. El análisis empírico muestra que la productividad científica está positivamente relacionada con la proporción de doctores, los vínculos o relaciones con otras instituciones para investigación y desarrollo y el financiamiento proveniente de instituciones científicas nacionales. Por tanto, la investigación sugiere un vínculo fuerte entre el capital social y las actividades de investigación.

\section{Cohesión -capital social bonding- y cooperación -capital social bridging-}

Teniendo en cuenta los postulados anteriormente descritos, en los grupos de investigación, desarrollo tecnológico e innovación el capital social es importante para la generación de sus resultados, a partir de sus dos dimensiones. Por un lado, la estructura interna (bonding), medido en grupos de investigación a través del indicador de cohesión. Por otra parte, la externa (bridging) medido en los grupos con el indicador de cooperación. Ministerio de Ciencia y Tecnología (2018) menciona que para cada grupo de investigación se calculan ambos indicadores, con el fin de evidenciar la existencia de trabajo mancomunado entre los integrantes del grupo y entre grupos. Para ello, se considera la coautoría, como una demostración clara de colaboración entre los autores. De esta manera, la cohesión hace referencia al valor que permite visibilizar la colaboración a nivel interno de los miembros del grupo. Este indicador se calcula de la siguiente manera:

$$
\mathrm{IC}=\frac{\text { Autores }}{\text { Productos }}-1
$$

Donde, "Autores" es el número total de autores del grupo relacionados en los productos de nuevo conocimiento y desarrollo tecnológico e innovación del grupo y "Productos" es el número de productos totales del grupo (Ministerio de Ciencia y Tecnología, 2018). La cooperación para cada grupo de investigación hace referencia al cálculo de su factor de colaboración, con el que se busca evidenciar el trabajo mancomunado entre grupos. De esta manera el indicador de cooperación es el valor que permite mostrar la colaboración, a partir de las coautorías declaradas en diferentes grupos. Este indicador se calcula de la siguiente manera:

$$
\text { ICoop }=\frac{\text { Número total de grupos relacionados }}{\text { Productos }}-1
$$

Donde, "Número total de grupos relacionados" son los grupos de investigación, desarrollo tecnológico o de innovación con los que están vinculados los coautores de los productos relacionados de nuevo conocimiento y desarrollo tecnológico e innovación del grupo y "Productos" es el número de productos del grupo (Ministerio de Ciencia y Tecnología, 2018). De esta manera, las conexiones entre los grupos a través de la cohesión y cooperación dan cuenta de la construcción colectiva de conocimiento, lo cual es clave para la generación de resultados de investigación. Estos espacios de interacción se convierten en apoyo fundamental para la creación y desarrollo de soluciones efectivas a problemáticas del entorno (Barrios-Hernandez et al., 2017)

\section{METODOLOGÍA}

En este artículo se plantea un tipo de investigación cualitativa, en la cual se realizó una revisión exhaustiva de la literatura sobre capital social y los resultados de los grupos de investigación en el sector de las IES, el cual ha sido poco estudiado desde esta perspectiva. Las fuentes consultadas en su mayoría fueron artículos científicos y documentos de bases de datos como Scielo, Scopus, Elseiver y Emerald de preferencia entre el año 2010 y 2020. Así también, se tomó en consideración publicaciones de fuentes gubernamentales como el Ministerio de Ciencias y Tecnología de Colombia. Para la captura de la información se desarrolló una revisión documental, tal como lo evidencia la Figura 2, inicialmente se realizó una búsqueda de la información con el fin de organizar las palabras clave y frases como capital social, capital social en el contexto universitario, integración social, perfil de colaboración de grupos de investigación, entre otras. Los documentos encontrados en bases de datos que se relacionen con el tema de la investigación han sido 53 documentos. Así mismo, se analizó el perfil de los grupos del Departamento del Atlántico publicado por el Ministerio de Ciencia y Tecnología en cuanto a los indicadores de cohesión y colaboración de los grupos para valorar el trabajo realizad con sus capacidades internas o a partir del relacionamiento con miembros de otros grupos de diferentes instituciones. Seguidamente se desarrolló una selección de documentos, para ello se clasificaron 
mediante una matriz que permitió valorar el concepto, la postura, los aspectos relevantes y principales autores. Posteriormente se eligen 41 documentos con mayor relevancia para la redacción del artículo. Además, se revisó el sistema de información del Ministerio de Ciencia y Tecnología y la clasificación de los grupos de investigación

Por último, se contrastó la información, tomando la información seleccionada para realizar reflexiones de acuerdo a las experiencias y observaciones previas que se tiene de los grupos de investigación y sus resultados descritos en el perfil de cada grupo. Adicionalmente se revisó la producción de los grupos con mayor indicador y las actividades más relevantes realizadas por estos.

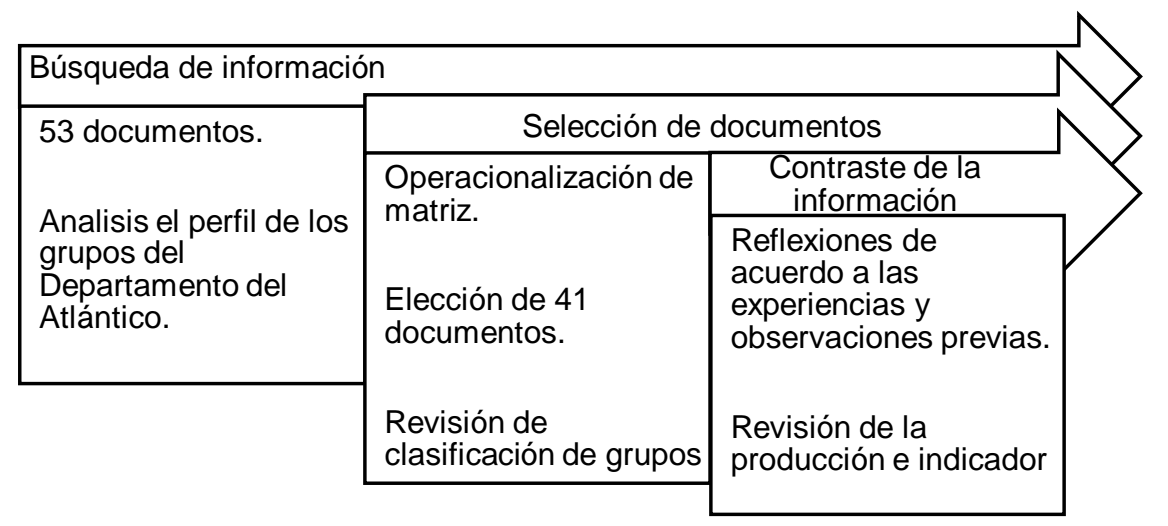

Fig. 2: Etapas de la revisión documental

\section{RESULTADOS}

El capital social ha tomado especial importancia en todas las organizaciones. De manera especial en las IES ha jugado un papel preponderante para alcanzar objetivos en el corto plazo, al aunar esfuerzo por unos mismos intereses. Los grupos de investigación, por su parte, han decido fortalecer sus relaciones de colaboración, no solo como algo propio del sistema, si como una manera de unir talentos por un mismo fin. De los 5539 grupos categorizados en la medición, 265 de ellos están ubicados en el Departamento del Atlántico, se encontró que 121 están categorizados en A1 o A, se obtuvo información sobre el perfil de colaboración de 114 de ellos, los cuales constituyen el centro de este estudio. Los 7 grupos restantes no tienen información reportada en la plataforma Scientic del Ministerio de Ciencia y Tecnología. Por tanto, no se pudo obtener información formal y validada de sus perfiles. Estos se ubican en 6 áreas de estudio como son las ciencias sociales, humanidades, ingeniería, ciencias de la salud y ciencias naturales. Los datos correspondientes con su perfil de cohesión y cooperación se presentan en la Tabla 2.

Tabla 2: Perfil de colaboración de grupos de investigación, desarrollo tecnológico e innovación. (Datos tomados del Ministerio de Ciencia y Tecnología, SBA. 2020)

\begin{tabular}{|c|c|c|c|}
\hline Área & Código del grupo & $I C$ & Icoop \\
\hline \multirow{17}{*}{ 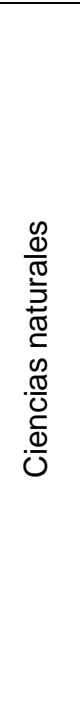 } & COL0010289 & 9.07 & 8.50 \\
\hline & COL0008129 & 19.57 & 10.50 \\
\hline & COL0005341 & 16.71 & 11.75 \\
\hline & COL0006329 & 10.50 & 34.50 \\
\hline & COL0000999 & 2.39 & 0.00 \\
\hline & COL0042999 & 9.55 & 25.50 \\
\hline & COL0050454 & 7.16 & 4.00 \\
\hline & COL0018045 & 9.55 & 17.50 \\
\hline & COL0054696 & 10.26 & 17.75 \\
\hline & COL0077064 & 27.21 & 30.00 \\
\hline & COL0015553 & 6.21 & 13.50 \\
\hline & COL0011329 & 39.62 & 20.25 \\
\hline & COL0095213 & 3.34 & 90.50 \\
\hline & COL0105599 & 15.99 & 26.75 \\
\hline & COL0128609 & 22.20 & 28.50 \\
\hline & COL0130475 & 2.86 & 6.00 \\
\hline & COL0171988 & 26.97 & 21.75 \\
\hline
\end{tabular}


Tabla 2 (continuación)

\begin{tabular}{|c|c|c|c|}
\hline Área & Código del grupo & $I C$ & Icoop \\
\hline \multirow{16}{*}{ 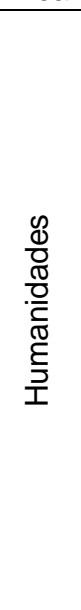 } & COL0008951 & 5.14 & 2.46 \\
\hline & COL0034156 & 13.14 & 0.00 \\
\hline & COL0036428 & 6.57 & 1.38 \\
\hline & COL0008853 & 9.43 & 0.77 \\
\hline & COL0010637 & 11.43 & 3.08 \\
\hline & COL0010519 & 6.00 & 0.00 \\
\hline & COL0042407 & 48.29 & 0.00 \\
\hline & COL0055076 & 19.14 & 0.46 \\
\hline & COL0083517 & 5.14 & 1.38 \\
\hline & COL0084612 & 88.00 & 0.08 \\
\hline & COL0013218 & 18.86 & 4.31 \\
\hline & COL0097488 & 32.86 & 0.69 \\
\hline & COL0098171 & 21.14 & 0.00 \\
\hline & COL0113367 & 13.14 & 4.85 \\
\hline & COL0114963 & 35.43 & 1.08 \\
\hline & COL0015615 & 54.57 & 2.46 \\
\hline \multirow{13}{*}{ 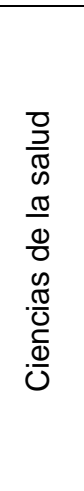 } & COL0021603 & 42.96 & 18.17 \\
\hline & COL0008933 & 9.93 & 1.83 \\
\hline & COL0038736 & 10.32 & 16.67 \\
\hline & COL0001781 & 13.63 & 34.5 \\
\hline & COL0060942 & 17.32 & 12.50 \\
\hline & COL0065312 & 21.02 & 5.67 \\
\hline & COL0070798 & 11.55 & 16.00 \\
\hline & COL0074465 & 8.78 & 25.83 \\
\hline & COL0015203 & 6.47 & 10.83 \\
\hline & COL0103683 & 0.92 & 29.17 \\
\hline & COL0113839 & 6.70 & 6.33 \\
\hline & COL0154637 & 7.16 & 6.33 \\
\hline & COL0183595 & 6.70 & 33.67 \\
\hline \multirow{24}{*}{ 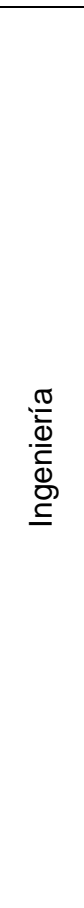 } & COL0012533 & 5.71 & 5.29 \\
\hline & COL0009968 & 14.29 & 2.71 \\
\hline & COL0016461 & 8.33 & 4.29 \\
\hline & COL0010996 & 15.00 & 11.29 \\
\hline & COL0016855 & 12.38 & 5.00 \\
\hline & COL0010904 & 14.05 & 10.00 \\
\hline & COL0038208 & 25.24 & 6.71 \\
\hline & COL0038594 & 28.10 & 47.86 \\
\hline & COL0008521 & 46.67 & 15.00 \\
\hline & COL0005501 & 7.62 & 12.71 \\
\hline & COL0040986 & 18.81 & 6.71 \\
\hline & COL0044902 & 25.95 & 7.00 \\
\hline & COL0045552 & 15.00 & 4.14 \\
\hline & COL0066848 & 11.43 & 27.57 \\
\hline & COL0070537 & 3.33 & 13.57 \\
\hline & COL0072809 & 13.10 & 15.71 \\
\hline & COL0074714 & 4.52 & 25.43 \\
\hline & COL0033945 & 38.57 & 6.71 \\
\hline & COL0067709 & 7.38 & 17.14 \\
\hline & COL0080097 & 30.24 & 37.71 \\
\hline & COL0087839 & 5.24 & 18.14 \\
\hline & COL0101301 & 21.19 & 7.14 \\
\hline & COL0012453 & 94.20 & 8.63 \\
\hline & COL0153783 & 14.98 & 44.09 \\
\hline \multirow{11}{*}{$\begin{array}{l}\mathscr{D} \\
\frac{0}{\pi} \\
\frac{\pi}{0} \\
0 \\
0 \\
\frac{.0}{0} \\
\frac{0}{0} \\
\frac{0}{0}\end{array}$} & COL0028032 & 6.79 & 10.14 \\
\hline & COL0029109 & 0.85 & 10.14 \\
\hline & COL0028838 & 5.31 & 4.71 \\
\hline & COL0012471 & 5.31 & 11.43 \\
\hline & COL0007696 & 6.79 & 5.00 \\
\hline & COL0014298 & 2.76 & 2.86 \\
\hline & COL0038629 & 14.44 & 9.43 \\
\hline & COL0039564 & 3.82 & 2.43 \\
\hline & COL0039591 & 15.92 & 19.14 \\
\hline & COL0007275 & 9.77 & 5.43 \\
\hline & COL0040832 & 9.34 & 9.00 \\
\hline
\end{tabular}


Tabla 2 (continuación)

\begin{tabular}{|c|c|c|c|}
\hline Área & Código del grupo & $I C$ & Icoop \\
\hline \multirow{34}{*}{$\begin{array}{l}\mathscr{D} \\
\frac{0}{\pi} \\
. \frac{\pi}{0} \\
0 \\
0 \\
0 \\
\frac{\pi}{0} \\
\frac{D}{0} \\
\frac{0}{0}\end{array}$} & COL0003229 & 9.77 & 2.86 \\
\hline & COL0004629 & 4.88 & 9.57 \\
\hline & COL0016962 & 27.18 & 1.57 \\
\hline & COL0001191 & 14.23 & 0.86 \\
\hline & COL0042194 & 10.40 & 22.29 \\
\hline & COL0043609 & 2.97 & 0.71 \\
\hline & COL0043585 & 9.34 & 8.00 \\
\hline & COL0046836 & 3.82 & 3.57 \\
\hline & COL0055109 & 4.88 & 26.71 \\
\hline & COL0056449 & 8.07 & 21.00 \\
\hline & COL0057259 & 2.34 & 8.14 \\
\hline & COL0067003 & 4.46 & 21.43 \\
\hline & COL0067335 & 11.46 & 15.71 \\
\hline & COL0068609 & 4.67 & 3.29 \\
\hline & COL0041061 & 9.98 & 13.86 \\
\hline & COL0075444 & 14.23 & 7.71 \\
\hline & COL0003229 & 9.77 & 2.86 \\
\hline & COL0076577 & 11.89 & 22.71 \\
\hline & COL0077019 & 11.46 & 23.57 \\
\hline & COL0027385 & 4.25 & 77.14 \\
\hline & COL0033364 & 0.00 & 20.00 \\
\hline & COL0011759 & 9.98 & 9.00 \\
\hline & COL0008693 & 6.79 & 10.43 \\
\hline & COL0012355 & 10.4 & 11.71 \\
\hline & COL0006269 & 19.32 & 9.71 \\
\hline & COL0088246 & 5.14 & 7.00 \\
\hline & COL0105928 & 18.26 & 21.43 \\
\hline & COL0114309 & 35.03 & 28.66 \\
\hline & COL0114711 & 3.61 & 17.14 \\
\hline & COL0137547 & 20.17 & 3.86 \\
\hline & COL0151223 & 15.50 & 22.43 \\
\hline & COL0153451 & 9.13 & 8.14 \\
\hline & COL0167386 & 2.97 & 18.86 \\
\hline & COL0170111 & 4.03 & 6.14 \\
\hline
\end{tabular}

A partir de la revisión de los datos anteriores, se analizaron las actividades más relevantes que realizaban los grupos. Poniendo especial atención en aquellos con un indicador de perfil de cohesión -capital social bondingy cooperación -capital social bridging- superior al 50\%. En la Tabla 3 se observa que para el caso de la cohesión se tienen 3 grupos con indicadores de 94.2, 88 y 54.7 respectivamente. El primero de ellos perteneciente al área de ingeniería, seguido de dos grupos de humanidades. De hecho, el grupo con mayor indicador de cohesión ocupa el puesto número 1 en desarrollo tecnológico en Colombia. Adicionalmente tiene como fortaleza su producción de artículos científicos, sin olvidar las actividades de consultoría, las cuales a partir de los resultados de medición de la convocatoria 2018 pasaron de ser clasificados de desarrollo tecnológico a apropiación social del conocimiento.

Tabla 3. Perfil de grupos de investigación con mayor indicador de cohesión -capital social bonding-

\begin{tabular}{|c|l|c|c|c|c|c|c|c|}
\hline \multirow{2}{*}{ Código } & \multirow{2}{*}{ Área } & \multirow{2}{*}{ IC } & \multicolumn{2}{|c|}{ Desarrollo tecnológico } & $\begin{array}{c}\text { Apropiación } \\
\text { social }\end{array}$ & \multicolumn{3}{c|}{ Nuevo conocimiento } \\
\cline { 4 - 11 } & & & $\begin{array}{c}\text { Innovación } \\
\text { de procesos }\end{array}$ & $\begin{array}{c}\text { Innovación } \\
\text { organizacional }\end{array}$ & Consultorías & Artículos & Libros & capítulos \\
\hline COL0012453 & Ingeniería & 94.20 & 40 & 30 & 203 & 78 & 13 & 58 \\
\hline COL0084612 & Humanidades & 88.00 & 1 & 7 & 14 & 14 & 23 & 7 \\
\hline COL0015615 & Humanidades & 54.57 & 0 & 16 & 1 & 8 & 0 & 1 \\
\hline
\end{tabular}

Los dos grupos siguientes, tienen sus productos divididos en las tres categorías. Sin embargo, se revisó que los tres tienen en común que la mayor parte de sus productos son desarrollados con miembros del mismo grupo. Al realizar un análisis de su producción científica en las categorías de desarrollo tecnológico, apropiación social y nuevo conocimiento, se observó que la fortaleza de estos grupos guarda disparidad en su producción. 
Los resultados obtenidos para estos grupos evidencian las dos posibles implicaciones del bonding capital cooperación- recogidas en la literatura. Por una parte, el ejemplo del grupo de ingeniería muestra que este grupo ha sido capaz de aprovechar la elevada cohesión entre sus miembros para obtener buenos resultados a nivel de desarrollo tecnológico, apropiación social y nuevo conocimiento. Sin embargo, los resultados obtenidos por los grupos de humanidades muestran los posibles inconvenientes recogidos en la literatura derivados de la elevada cohesión entre sus miembros, como pueden ser el bloqueo, y que lleva a estos grupos a obtener bajos resultados a nivel de desarrollo tecnológico, apropiación social y nuevo conocimiento (Eklinder-Frick et al., 2012).

Por otro lado, para el caso de perfil de cooperación en la Tabla 4 se evidencia que un grupo ciencias naturales tiene un indicador de 90,5 y otro perteneciente a las ciencias sociales posee un indicador de 77,14 . Al analizar sus actividades se observó que contrario a los grupos con mayor indicador de cohesión, estos tienen en común su fortaleza en la producción de nuevo conocimiento, a través de la publicación de artículos en revistas indexadas cuyos trabajos son realizados por miembros de diferentes grupos. En este caso, los resultados concuerdan con los planteamientos recogidos en la literatura que establecen una influencia positiva del capital social bridging -cooperación- en el planteamiento de nuevas ideas y desarrollo de innovaciones, que en este caso se traduce en unos buenos resultados en desarrollo tecnológico, apropiación social y nuevo conocimiento (Doloreux y Mattson 2008).

En este sentido, la cooperación en estos grupos de investigación se relaciona con la necesidad de complementar capacidades, debido a que la realizacion de proyectos deriven luego en una producción cientifica generalmente de alto impacto y esta requiere la cooperacion de actores multidisciplinares que permita realizar un abordaje mas integral. Este indicador, se explica tambien por los beneficios que los grupos obtienen por el reconocimiento de la sinergias que ofrecen sus interacciones en las politicas de las propias IES, quienes actualmente en Colombia muestran un interes creciente en que se desarrollen productos con grupos externos y en la consecución de financiación a partir de la generación de alianzas.

Tabla 4. Perfil de grupos de investigación con mayor indicador de cooperación -capital social bridging-

\begin{tabular}{|c|c|c|c|c|c|c|c|c|}
\hline \multirow{2}{*}{ Código } & \multirow{2}{*}{ Área } & \multirow{2}{*}{ I Coop } & \multicolumn{2}{|c|}{ Desarrollo tecnológico } & \multicolumn{2}{c|}{$\begin{array}{c}\text { Apropiación } \\
\text { social }\end{array}$} & \multicolumn{2}{c|}{ Nuevo conocimiento } \\
\cline { 4 - 9 } & & $\begin{array}{c}\text { Innovación } \\
\text { procesos }\end{array}$ & $\begin{array}{c}\text { Innovación } \\
\text { organizacional }\end{array}$ & Consultorías & Artículos & Libros & capítulos \\
\hline COL0095213 & $\begin{array}{l}\text { Ciencias } \\
\text { Naturales }\end{array}$ & 90.50 & 0 & 1 & 1 & 88 & 1 & 5 \\
\hline COL0027385 & $\begin{array}{l}\text { Ciencias } \\
\text { Sociales }\end{array}$ & 77.14 & 2 & 1 & 3 & 82 & 13 & 19 \\
\hline
\end{tabular}

Adicionalmente el grupo con mayor indicador de cooperación está conformado por científicos del área de las ciencias naturales, especialmente la genética humana, este grupo cuenta con su propio laboratorio de virología y con un programa de doctorado y maestría en genética, allí convergen el desarrollo de diferentes investigaciones en las cuales colaboran integrantes de grupos externos.

Toda la información anterior permitió evidenciar que las áreas de estudio ingeniería, humanidades, ciencias naturales, ciencias de la salud, ciencias sociales muestran disparidad en sus resultados entre áreas y dentro de ellas. Debido a que el papel del investigador juega un papel fundamental para el desarrollo de relaciones internas y externas. Además, el indicador de cohesión total tiende a ser más alto en los grupos pertenecientes a las ciencias naturales, humanidades e ingeniería por la naturaleza misma de estos grupos quienes se agrupan internamente para fortalecer sus investigaciones y desarrollar productos en conjuntos. Mientras para el caso del indicador de cooperación se observa, que éste guarda un comportamiento similar para las cinco áreas. No existe una tendencia significativa en un área en particular. Lo anterior debido a que internamente las IES están promoviendo el desarrollo de trabajos entre pares de diferentes grupos ajenos a la institución, con el fin de no solo fortalecer la investigación propiamente dicha, si no de estrechar relaciones de valor con miembros externos a la institución.

A partir de los resultados obtenidos puede evidenciarse que los miembros de los grupos pueden establecer relaciones de cooperación con miembros externos de otros grupos que les permitan identificar nuevas ideas y conocimiento complementario para el desarrollo de sus investigaciones. Por otra parte, se debe continuar aprovechando el conocimiento de sus redes internas con precaución, intentando evitar la excesiva frecuencia y estrechez en las mismas que pueda llevar a bloqueos en la información. 


\section{CONCLUSIONES}

Siendo las IES instituciones generadoras de nuevo conocimiento, parte de sus resultados se ven representados por la relación interna y externa de los grupos de investigación, desarrollo tecnológico e innovación. El análisis del capital social de estos grupos a partir del perfil de colaboración para los indicadores de cohesión -capital social bonding- y cooperación -capital social bridging-, permitió extraer las siguientes cuatro conclusiones principales:

1) El capital social está afectado con el valor que cada miembro de un grupo les da a sus relaciones con otros, a través de los intereses personales, la confianza y las redes. Por ende, estas relaciones tienen una reciprocidad de alto valor que permiten fortalecer las relaciones.

2) Una característica de los grupos de investigación es que se conciben como organizaciones sociales que facilitan y fortalecen su trabajo en la medida que establecen conexiones internas de cohesión o externas de cooperación. Por tanto, la interacción social de los grupos de investigación se desarrolla como un vínculo fuerte, lo que se convierte en un determinante clave para el intercambio de conocimiento y la generación de resultados de investigación en conjunto, que permite no solo una buena categorización sino el desarrollo de capital social de alto valor.

3) Debido al mismo sistema de medición de grupos de investigación, desarrollo tecnológico e innovación en Colombia, desde el nivel corporativo de las IES se establecen estrategias de relacionamiento de sus investigadores con sus pares de la misma institución o de otras externas, con el fin de fortalecer el aprendizaje e incrementar el nivel del conocimiento como el activo intangible más importante de este tipo de instituciones, en paralelo a responder a las necesidades del entono. 4) Igualmente, se evidencia que los grupos de investigación establecen internamente su estrategia de capital social. En este sentido que observa, que el fortalecimiento en sus relaciones de cohesión conlleva resultados divergentes, emergiendo para un grupo las implicaciones positivas del capital social bonding, lo que lleva a lograr mejores resultados, y por otra los aspectos negativos para otros grupos, que los llevan a obtener niveles bajos de resultados (Eklinder-Frick et al., 2012).

Por otra parte, otros grupos favorecen su relacionamiento a través de la cooperación con grupos externos capital social bridging-y en este sentido también logran los resultados esperados (Doloreux y Mattson 2008). Lo que si es cierto para el caso de esta investigación es que son escasos los grupos que desarrollan su capital social a través del bonding y bridging simultáneamente.

\section{REFERENCIAS}

Arzuero, A. Capital social e inclusión social. Cuadernos de Administración, Vol 25 (41) 151-168 (2009).

Barletta, F., Yoguel, G., Pereira, M., y Rodriguez, S. Exploring scientific productivity and transfer activities: Evidence from Argentinean ICT research groups. Research Policy. Vol 46(8), 1361-1369 (2017).

Barrios-Hernández, K., y Olivero-Vega. E. Relación universidad-empresa-estado. Un análisis desde las instituciones de educación superior de Barranquilla-Colombia, para el desarrollo de su capacidad de innovación. http://dx.doi.org/10.4067/S0718-50062020000200021. Formación Universitaria Vol. 13(2), 21-28 (2020)

Barrios-Hernández, K., Olivero-Vega. E. Y Figueroa-Saumet, B. Condiciones de la gestión del talent humano que favorecen el desarrollo de las caapcidades dinámicas. http://dx.doi.org/10.4067/S0718-07642020000200055. Información. Tecnológica Vol. 31(2), 55-62 (2020)

Barrios-Hernández, K., Olivero-Vega, E. y Acosta-Prado, J. Capacidad dinámica de innovación en instituciones de educación superior. Revista Espacios. Vol. 38 (1), 24 (2017).

Barrios-Hernández, K., Olivero Vega, E. y otros veintitrés autores. Gestión del conocimiento y capacidad de innovación. Modelos, Sistemas y Aplicaciones. Barranquilla: Universidad Simón Bolívar (2017).

Bourdieu, P. y Wacquant, L. An Invitation to Reflexive Sociology, University of Chicago Press, Chicago(1992).

Bourdieu, P. Le capital social. Notes provisoires. Actes de la Recherche en Sciences Sociales, 31, 2-3 (1980)

Burt, R. Structural Holes: The Social Structure of Competition. Cambridge. Hardvard University Press (1992).

Capdevielle, J. Capital social: debates y reflexiones en torno a un concepto polémico. http://dx.doi.org/ 10.1590/1678987314225101. Revista de Sociología e Política Vol. 51, 3-14 (2014).

Clark, B. Sustaining Change in Universities, Society for Research into Higher Education. Londres: Open University Press (2004).

Coleman, J. S. Social Capital in the Creation of Human Capital. American Journal of Sociology 94:95-120 (1988).

Doloreux, D., y H. Mattson. To What Extent Do Sectors Socialize Innovation Differently? Mapping Cooperative Linkages in Knowledge-Intensive Industries in the Ottawa Region. Industry \& Innovation 15 (4): 351-70 (2008). 
Dyer, J. y Nobeoka, K. Creating and Managing a High-Performance Knowledge Sharing Network: The Toyota Case http://dx.doi.org/10.1002/(SICl)1097-0266(200003). Strategic Management Journal, 21, 345-367. $21(2000)$

Eklinder-Frick, J., L. Torsten Eriksson, y L. Hallen. Effects of Social Capital on Processes in a Regional Strategic Network. Industrial Marketing Management 41 (5): 800-6 (2012).

Elche-Hortelanoa, D., Martínez-Péreza, A., y García-Villaverde, P. Bonding. Capital, explotación de conocimiento e innovación incremental en los clusters de turismo cultural: las Ciudades Patrimonio de la Humanidad en España. Investigaciones Europeas de Dirección y Economía de la Empresa, 21(3), 120-128 (2015).

Esparcia, J., Escribana, J., y Serrano, J. Una aproximación al enfoque del capital social y a su contribución al estudio de los procesos de desarrollo local. Investigaciones Regionales (34), 1-10 (2015).

Freyre, M. El capital social. Alcances teóricos y su aplicación empírica en el análisis de políticas públicas. Revista Ciencia, Docencia y Tecnología de la Universidad Nacional de Entre Ríos 24(47), 95-118 (2013).

Forni, P., Siles, M. y Barreiro, L. Qué es el Capital Social y cómo analizarlo en contextos de exclusión y pobreza: Estudios de caso en Buenos Aires, Argentina. JSRI Research Report (35). The Julian Samora Research Institute, Michigan State University, East Lansing, Michigan (2004).

Gaette, J. un aporte al enfoque de redes sociales en el campo de la educación superior, a partir de la identificación y análisis de la (s) red(es) de conocimiento recreada(s) Revista hispana para el análisis de redes sociales, 14(5), 1-33 (2008).

Hanifan, L. J. The Rural School Community Center, Annals of the American Academy of Political and Social Science, 67,130-138 (1916).

Kale, P., Singh, H. y Perlmutter, H. Learning and Protection of Proprietary Assets in Strategic Alliances: Building Relational Capital, Strategic Management Journal, 21, 217-237(2000).

Kyvik, S., y Reymert, I. Research collaboration in groups and networks: differences across academic fields. Scientometrics (113), 951-957 (2017).

Lozares, C., López Roldán, P. y otros tres autores. Cohesión, Vinculación e Integración sociales en el marco del Capital Social», REDES - Revista hispana para el análisis de las redes sociales, 20 (1), 1-28 (2011).

Martínez, A. Capital social e innovación en los clústers de turismo cultural: el efecto mediador de la estrategia de conocimiento (Tesis Doctoral). Cuenca, España: Universidad Castilla La Mancha (2013).

Ministerio de Ciencia y Tecnología. Modelo de medición de reconocimientos de grupos de investigación, desarrollo tecnológico e innovación y de reconocimiento de investigadores del sistema nacional de ciencia, tecnología e innovación. (2018).

Ministerio de Ciencia y Tecnología. Publicación de resultados finales de la convocatoria 833 de 2018. Bogotá: Ministerio de Ciencia y Tecnología. (2019).

Ministerio de Ciencia y Tecnología. Sistema de información. Bogotá: Ministerio de Ciencia y Tecnología (2020).

Nahapiet, J., y S. Ghoshal. Social Capital, Intellectual Capital, and the Organizational Advantage. Academy of Management Review 23 (2): 242-66 (1998).

Orozco, L.A., Ruiz, C.F., Bonilla, R. y Chavarro D.A. Los grupos de investigación en Colombia. Sus practicas, su reconocimiento y su legitimidad. En M. Salazar y L. Fog (eds.) (2013).

Putnam R.D. Bowling Alone. The Collapse and Revival of American Community. Simon and Schuster, Nueva York(2000).

Rostila, M., y Saarela, J. M. (2011). Time does not heal all wounds: Mortality following the death of a parent. https://doi.org/10.1111/j.1741-3737.2010.00801.x 73. Journal of Marriage and Family. (1), 236-249 (2011).

Segura, J. Capital social vínculo ("Bonding”) y Capital social puente (“Bridging”) (2011).

Steinmo, M., y Rasmussen, R. The interplay of cognitive and relational social capital dimensions in university-industry collaboration: Overcoming the experience barrier. Research Policy, 41(10), 1964-1974 (2018).

Solis, F., y Limas, M. Capital social y desarrollo: origen, definiciones y dimensiones de análisis. Nóesis. Revista de Ciencias Sociales y Humanidades, 22(43-1), 187-212 (2013).

Tyndall, D., Forbes, T., Avery, J., y Powell, S. Fostering scholarship in doctoral education: Using a social capital T framework to support PhD student writing groups. Journal of Professional Nursing, 35(4), 300-304 (2019).

Walter, J., Lechner, C. y Kellermanns, L. Journal of Business Research. Vol. 60, issue 7, 698-710 (2007).

Westlund, H. Social Capital in the Knowledge Economy: Theory and Empirics, New York, NY, Springer-Verlag (2006). 